\title{
Initial Approach to Collect Small Unmanned Aircraft System Off-nominal Operational Situations Data
}

\author{
Jaewoo Jung* \\ NASA Ames Research Center, Moffett Field, CA 94035 \\ Charles R. Drew ${ }^{\dagger}$ \\ Wyle Labs, Moffett Field, CA 94035 \\ Sreeja Nag\$ \\ Bay Area Environmental Research Institute, Moffett Field, CA 94035 \\ Edgar O. Torres ${ }^{\S}$ \\ Science Application International Corp., Moffett Field, CA 94035 \\ Abraham K. Ishihara**, Minh Do ${ }^{\dagger \dagger}$ \\ Stinger Ghaffarian Technologies, Inc. Moffett Field, CA 94035 \\ and \\ Hemil C. Modi \\ Science and Technology Corp., Moffett Field, CA 94035
}

\begin{abstract}
NASA is developing the Unmanned Aircraft System Traffic Management research platform to safely integrate small unmanned aircraft operations in large-scale at low-altitudes. As a part of this effort, small unmanned aircraft system off-nominal operational situations data collection process has been developed to take lessons learned and to reinforce operational compliance. In this paper, descriptions of variables used for digital data collection and an online report form for collection of observational data from the operators (contextual data) are provided. They are used to collect off-nominal data from the Unmanned Aircraft System Traffic Management National Campaign in 2017. The digital data show that 2 out of 118 campaign operations (1.7\%) encountered loss of navigation. Since the campaign aircraft used Global Positioning System for navigation, it is likely that unobstructed view of the sky at the campaign locations contributed to this small number. Also, 4 out of 47 operations (8.5\%) encountered loss of communications. A relatively short distance between ground control system and aircraft, ranging from 2300 feet to 4200 feet, likely contributed to this small number. There was no data to identify the loss of communications condition, aircraft received signal strength, for the remaining 71 operations suggesting that some operators may not be monitoring unmanned aircraft communications system performance or monitoring it with different parameters. For the contextual data, due to the low number of total reports during the campaign, no significant trends emerged. This is an initial attempt to collect contextual data from small unmanned aircraft operators about off-nominal situations, and changes will be made to the future data collection to improve the amount and quality of the information.
\end{abstract}

\footnotetext{
* Aerospace Technologist, Aerospace High Density Operations Branch, Mail Stop 210-6. Senior Member, AIAA.

$\dagger$ Captain, ASRS Program Manager (ret.), NASA Mission Specialist, UTM, Mail Stop 210-6.

* Research Engineer, Mail Stop 210-6. Member, AIAA.

$\S$ UTM Engineer, Mail Stop 210-15.

** Research Scientist, Mail Stop 210-6. Member, AIAA.

$\dagger$ Senior Research Scientist, Planning and Scheduling Group, Mail Stop 269-3.

$\$$ Aerospace Research Scientist, Mail Stop 245-3.
} 


\section{Introduction}

As of December 2016, there were more than 670,000 registered small Unmanned Aircraft System (UAS) in the United States, 626,000 as hobbyists $\$$ and 44,000 as commercial. These numbers are expected to grow rapidly over the coming years [1]. Operations of these aircraft beyond hobby or recreation in the U.S. is currently regulated by Federal Aviation Regulation part 107 (Part 107), and numerous Visual Line of Sight (VLOS) applications including aerial photography, real estate, construction, industrial and utility inspection and agriculture are enabled under this regulation [2]. Operations beyond what is allowed under Part 107, such as Beyond Visual Line of Sight (BVLOS) operations, operations over people, and night operations, are enabled through the Federal Aviation Administration (FAA)'s waiver process [3]. Whereas the FAA strives to review and issue decisions within 90 days in this process, a limited number of waivers are granted, as there is no established infrastructure to integrate large-scale beyond-Part 107 operations into the National Airspace System (NAS) without endangering people or property on the ground or in the air.

To safely and efficiently integrate the full gamut of small UAS operations at low altitudes, NASA has developed the UAS Traffic Management platform $[4,5]$ to research and develop promising technologies and data exchange protocols to support routine and widespread execution of present and future envisioned applications such as urban area package delivery and BVLOS infrastructure inspection, and also to perform conceptual and technical research that can be transferred to the FAA in the form of airspace integration requirements for further testing. For this transfer, NASA and the FAA formed the UTM Research Transition Team (RTT) with goals to research and mature increasingly complex UTM operational scenarios and technologies; demonstrate those capabilities on the NASA UTM research platform; and deliver to the FAA technology transfer packages that enable NAS service expectations for low-altitude airspace operations by providing insight and capability requirements for critical services [6].

Given the wide technological areas and operating environments that the UTM RTT is addressing, the team has been divided into four focus subgroups, Concepts and Use Cases (CWG); Data Exchange and Information Architecture (DWG); Sense and Avoid (SAA); and Communications and Navigation (C\&N). The CWG is providing the conceptual framework, scenarios, and use cases for the other subgroups to explore. The DWG works to identify, develop, and test expected data exchanges and architectural implications and challenges. The SAA and C\&N work to identify and evaluate key performance and operations challenges and constraints. Outputs from the DWG, the SAA, and the $\mathrm{C} \& \mathrm{~N}$ subgroups in turn inform the CWG to progressively elaborate the UTM concept of operations.

This paper describes a part of $\mathrm{C} \& \mathrm{~N}$ activities where the small UAS off-nominal operational situations data collection process has been developed to gain lessons learned, and to reinforce operational compliance to ensure that the Unmanned Aircraft (UA) is under operational control of the pilot and remains within a defined area. Specifically, descriptions of variables for digital data collection and an online report form for collection of contextual data, defined in this paper as observational data from the UAS operators, are provided. These variables and form were used to collect off-nominal data from the UTM National Campaign in 2017, and two off-nominal operational events, loss of navigation and loss of command and control (C2) link are examined using the digital data. Additionally, contextual reports collected via the online form are used to investigate circumstances of the off-nominal events and actions taken by the operators. The paper is concluded with discussion on limitations found from this initial off-nominal data collection approach and the next steps.

\section{Brief Description of UTM TCL2 National Campaign}

In 2017, NASA contracted with six FAA UAS Test Sites [7] to demonstrate the UTM capability at that time, Technical Capability Level 2 (TCL2), that supports VLOS and BVLOS operations over sparsely populated land and where there are few manned aircraft in close proximity to the UA operations area. The goals of this demonstration, known as the UTM TCL2 National Campaign (NC II), are to test scenarios across a wide range of UAS platforms and locations across the U.S., and to utilize then-recently-developed UAS Service Supplier (USS) and Flight Information Management System (FIMS) architecture to validate further the scalability of the UTM concept and architecture. To achieve these goals, NASA guided the Test Sites to design and execute flight operations to meet one or more of the following objectives.

1. Conduct operations with a TCL 2-compatible USS that was not developed by NASA

2. Conduct operations to test and determine information requirements between the components of the UTM platform

3. Test USS and human operator reactions to simulated Air Navigation Service Provider constraints and directives

4. Test and determine weather service information requirements

$\S \S$ Owner-hobbyist can register once and apply the same registration number to multiple aircraft. Therefore, there can be more UAS than the number of registrations. 
5. Test surveillance services

6. Test and evaluate scheduling and planning capabilities

7. Evaluate best practices for ground-based sense and avoid

8. Evaluate geofencing and conformance monitoring capabilities

9. Gather data to develop communication and navigation guidance to industry to ensure that 1) UA are under operational control of the pilot, and 2) UA remain within a defined area

10. Evaluate human factors requirements related to data creation and display

11. Demonstrate a BVLOS package delivery while other aircraft are operating nearby.

The NC II flight operations took place from May $15^{\text {th }}$ to June $9^{\text {th }}$, and data from these operations were captured for analysis. The participating FAA test sites, test locations, test range descriptions, vehicle types and USS providers are shown in Table 1. Appendix A shows the FIMS-USS architecture used in the NC II. Further information about the NC II, such as test scenarios for each test site and discussion on measure of performance, can be found in [8].

Table 1. UTM National Campaign Test Sites

\begin{tabular}{|c|c|c|c|c|}
\hline $\begin{array}{l}\text { FAA Test } \\
\text { Site }\end{array}$ & Test Range Location & Range Description & Vehicle Type & $\begin{array}{l}\text { USS } \\
\text { Provider }\end{array}$ \\
\hline Alaska & $\begin{array}{l}\text { Three locations at the Poker } \\
\text { Flat Research Range, and the } \\
4^{\text {th }} \text { at Fairbanks. }\end{array}$ & $\begin{array}{l}\text { The test area is sub-arctic with scrub and } \\
\text { trees. The Fairbanks location is an urban } \\
\text { area. }\end{array}$ & $\begin{array}{l}1 \text { helicopter } \\
3 \text { quadcopter } \\
2 \text { octocopter }\end{array}$ & Simulyze \\
\hline $\begin{array}{l}\text { North } \\
\text { Dakota }\end{array}$ & $\begin{array}{l}11 \text { miles south of Grand } \\
\text { Forks, ND and } 1.5 \text { miles east } \\
\text { of Thompson, ND }\end{array}$ & Farmland with an above-ground powerline. & $\begin{array}{l}1 \text { hybrid } \\
4 \text { fixed wing } \\
2 \text { hexacopter } \\
1 \text { simulated }\end{array}$ & Simulyze \\
\hline Nevada & Reno-Stead Airport & $\begin{array}{l}\text { Much of the range was used for the TCL } 2 \\
\text { Demonstration in October } 2016 \text {. The range } \\
\text { was extended north to accommodate long } \\
\text { range flights. }\end{array}$ & $\begin{array}{l}4 \text { fixed wing } \\
2 \text { quadcopter } \\
1 \text { hexacopter } \\
1 \text { octocopter }\end{array}$ & $\begin{array}{l}\text { AirMap, } \\
\text { Amazon }\end{array}$ \\
\hline New York & Griffiss International Airport & $\begin{array}{l}\text { Lies within Griffiss Class D airspace. } \\
\text { Includes an area of about } 220 \text { acres where } \\
\text { airborne UAS can gather to avoid manned } \\
\text { traffic. }\end{array}$ & $\begin{array}{l}1 \text { quadcopter } \\
1 \text { hexacopter } \\
1 \text { octocopter } \\
1 \text { hybrid }\end{array}$ & NASA \\
\hline Texas & $\begin{array}{l}\text { Laguna Test Range in Port } \\
\text { Mansfield, TX }\end{array}$ & $\begin{array}{l}\text { Coastal range including Charles R. Johnson } \\
\text { Airfield. Flights were conducted over both } \\
\text { ground and water (i.e., the Gulf of Mexico). }\end{array}$ & $\begin{array}{l}2 \text { quadcopter } \\
2 \text { fixed wing }\end{array}$ & NASA \\
\hline Virginia & $\begin{array}{l}\text { Kentland Farm Agricultural } \\
\text { Research Center, Blacksburg, } \\
\text { VA }\end{array}$ & $\begin{array}{l}\text { Owned by Virginia Tech. } 1,800 \text { acres in size, } \\
\text { bordered on the SW by the New River. } \\
\text { Includes an asphalt airstrip. }\end{array}$ & $\begin{array}{l}1 \text { fixed wing } \\
2 \text { quadcopter }\end{array}$ & $\begin{array}{l}\text { Google, } \\
\text { ANRA }\end{array}$ \\
\hline
\end{tabular}

\section{Description of Off-nominal Data Collection Approaches}

\section{A. Variables for Digital Off-nominal Data Collection}

The NC II Data Management Plan (DMP) consists of four data sets; Aircraft Flight Plan; UAS specifications; UAS State; and Auxiliary UAS Operation. Several variables were added to the last three sets to collect off-nominal operational situation data.

1. Aircraft Flight Plan

The first set of the data compendium is an Aircraft Flight Plan, which specifies the date and time of the flight, UAS Vehicle Identification Number (UVIN), which is the vehicle identification number for a single aircraft that is obtained from a prototype NASA USS, Globally Unique Flight Identifier (GUFI), which is the individual flight identification number generated by a USS, waypoint location (Latitude/Longitude/Altitude), target air and ground speeds, hover time and a timestamp based on the Global Positioning System (GPS) week/second standard. Additionally, an "as run" version of a flight test card or procedure was used by the test participant during the execution of the test for archiving and analysis.

2. UAS Specifications

Specifications for each UAS used in the flight test are collected in this set. These include UAS maker/model, UVIN, and aircraft characteristics such as dimensions, and performance and Ground Control System (GCS) 
information. For off-nominal data collection, variables to capture communication system sensitivity, communications protocol, navigation system, navigation solution errors and others are added to this set.

\section{UAS State}

UAS State data refers to time-dependent data taken during flight. Each data point is tagged with UVIN, GUFI and the date \& time of the flight in GPS time. Collected data includes real-time position (Latitude/Longitude/Altitude) and velocity of the vehicle, sensor information, attitude, motor conditions, actuator commands, battery status, target waypoints and aircraft airborne state. For off-nominal operation situation analysis, types of data collected by the Aviation Safety Information Analysis and Sharing (ASIAS) system $[10,11]$ and UAS operation specific data types, such as C2 link Received Signal Strength Indicator (RSSI) measured at aircraft and GCS, are added to this set.

4. Auxiliary UAS Operation

This set encompassed all the time-independent data that was not part of the Flight Plan or uniquely related to the UAS Specifications. Operators provided UVIN, GUFI, type of operation (e.g. "live" or "simulated"), airframe, flight test card, takeoff weight and location, and landing location. For off-nominal data collection, GCS location variables are added to this set.

A NASA-hosted website ${ }^{* * *}$ was created to intake the DMP-compliance data. Appendix B shows all DMP variables that were used to collect data from the NC II operations, where off-nominal data variables' names are highlighted in bold typeface.

\section{B. Online Report Form for Contextual Data}

To collect contextual data, an online form was developed based on a general report form used by the Aviation Safety Reporting System (ASRS) [11-13]. ASRS uses its form to gather details and background information about commercial Aviation incidents and accidents, and it was determined that a similar form can be used to gather information about small UAS off-nominal operational situations. Appendix C shows the questions in the NC II online form. During the NC II, UAS operators were asked to complete the form if any of the following events occurred: a loss or degradation of communication between the vehicle and the GCS; a loss or degradation of any navigation signal, or any other navigation system failure; any lateral or vertical excursion from planned flight geography; and any loss of vehicle control, including propulsion system, flight control system, or vehicle structural problems or failures. Completing the form was entirely voluntary.

\section{The NC II Off-Nominal Data Analysis Results}

More than 1700 operation plans were submitted in real-time to the USS during the NC II. Since these plans included ground tests and shakedown flights, significant filtering effort involving manual and automated processes were made to identify 118 data collection flights. The off-nominal situation investigation was limited to these flights. The collected digital data were used to investigate loss of navigation and loss of C2 link off-nominal situations. The collected contextual data was evaluated by an ASRS Subject Matter Expert to better understand off-nominal situations.

\section{A. Loss of Navigation}

From the NC II digital data, the number of GPS satellites tracked by the GPS receiver in the navigation system onboard UA, represented as a variable "numGpsSat_nonDim," is used to reflect the loss of navigation off-nominal operational situation. This off-nominal situation is detected when the aircraft navigation system tracks six or fewer satellites for ten seconds or more. The six-satellite threshold number was selected, as lower than the baseline for ideal operating conditions for GPS-based navigation in open area with an unobstructed view of the sky. The threshold time of ten seconds was selected based on the UAS community input. The data show that only 2 out of 118 campaign operations $(1.7 \%)$ encountered loss of navigation. Since the campaign aircraft used GPS for navigation, it is likely that the unobstructed view of the sky at the campaign locations, described in Table 1, contributed to this small incidence of loss of navigation. It is expected that operations in areas where line of sight to GPS satellites can be easily blocked, such as an urban canyon, would experience an increase in loss of navigation if GPS is the sole means of navigation and the same formula is used to detect loss of navigation. To address this issue, for operations in urban area UA should be equipped with navigation systems that can rely on other sensors, such as Light Detection and Ranging (Lidar) and radar, to cope with lost line of sight to GPS satellites.

\section{B. Loss of Command and Control Link}

\footnotetext{
${ }^{* * *}$ http://utmregistry.arc.nasa.gov/ requires a credential from NASA to access.
} 
The $\mathrm{C} 2$ radio link RSSI measured at the aircraft in $\mathrm{dBm}$, represented as a variable "c2RssiAircraft_dBm," is used to reflect the loss of $\mathrm{C} 2$ link. This off-nominal situation is detected when the RSSI remains at or below -90dBm for more than 10 seconds. A value of $-90 \mathrm{dBm}$ is a typical radio-received sensitivity derived from UAS specifications. The data shows that 4 out of these 47 operations $(8.5 \%)$ encountered actual loss of $\mathrm{C} 2$ link. This indicates that the communications systems used in the NC II, such as $900 \mathrm{MHz}$ and $2.4 \mathrm{GHz}$ radios, provided sufficient performance to cover relatively short distance between the GCS and aircraft, ranging from 2300 feet to 4200 feet. Also, unobstructed radio line of sight between UA and the GCS likely contributed to this small number of incidence of loss of C2 link.

Data necessary to identify loss of C2 link situation was only received for 47 out of 118 operations (39.8\%). This lack of data for the remaining 71 operations suggests that some operators may not be monitoring communications system performance during operation, may not be aware of which variables to monitor, or may be monitoring communications system performance with a variable different from the C2 RSSI received by the aircraft. To address the first two conditions, further engagement with the UAS operator community is needed to encourage communications system performance monitoring. To address the last condition, developing a de-facto standard to monitor communications systems is needed.

\section{Contextual Data}

From the NC II, 15 non-duplicative reports of contextual data were received via online form in response to various events. Of these, 9 originated from the Remote-Control Pilot, 4 from the GCS Operator, 1 from a Mission Manager and 1 from a Flight Engineer. Table 2 provides Event Type and Counts. Note that multiple responses were permitted, thus respondents could provide more than 1 response to a single event; 4 reports represented more than 1 event type. Table 3 summarizes the Type of Loss of Control encountered. Table 4 summarizes the Action Taken and Result.

Table 2. Events and Counts

\begin{tabular}{|l|c|}
\hline \multicolumn{1}{|c|}{ Event } & Count \\
\hline Other navigation system failure & 4 \\
\hline Loss/Degradation of GCS to vehicle communication & 3 \\
\hline Loss/Degradation of vehicle to GCS communication & 3 \\
\hline GPS or other navigation system signal loss/degradation & 2 \\
\hline Vertical Deviation from flight geography & 2 \\
\hline Lateral Deviation from flight geography & 0 \\
\hline & $\mathbf{1 4}$ \\
\hline
\end{tabular}

Table 3. Type of Loss of Control

\begin{tabular}{|c|c|}
\hline Type of Loss of Control & Count \\
\hline Other (please specify) & 7 \\
\hline Flight Control System failure & 3 \\
\hline Motor failure & 1 \\
\hline Power (battery) failure & 0 \\
\hline Propeller Failure & 0 \\
\hline Structural failure & 0 \\
\hline Total & 11 \\
\hline
\end{tabular}

Table 4. Action Taken and Result

\begin{tabular}{|l|c|}
\hline Action Taken & Count \\
\hline Other (please specify) & 5 \\
\hline Land In Place & 3 \\
\hline Return To Base (Return To Launch Site) & 2 \\
\hline Terminate & 1 \\
\hline Vehicle impacted terrain & 0 \\
\hline Flight Returned to Planned Track or Heading & 0 \\
\hline Loiter & 0 \\
\hline None & 0 \\
\hline Regained Aircraft Control & 0 \\
\hline Regained Communication & 0 \\
\hline Regained Navigation Capability & 0 \\
\hline Return to flight geography (authorized airspace) & 0 \\
\hline Vehicle lost & $\mathbf{1 3}$ \\
\hline Total & \\
\hline
\end{tabular}


Of the 14 contextual reports of off-nominal conditions that included wind-direction and speed data, only 3 were 10 knots or above, specifically 10,11, and 12 knots, and all speed values were within the performance envelope of the associated UA. Therefore, wind velocity was not considered a significant factor in these incidents. Of the 13 reports providing density altitude data, 2 reports stated that density altitude was "unknown" and one report indicated density altitude in excess of 6,000 feet MSL. The remainder reported density altitude between 504 feet to 2696 feet. From this data, it was determined that density altitude did not play a significant role in precipitating off-nominal failures specified here. The distribution of events among aircraft types was unremarkable, and no question responses or categories indicated an unusual distribution of event or results. Due to the low number of reports, no significant trends emerged. With the small amount of available data, it is uncertain what might be potential underlying common contributors to off-nominal situations. The test team has identified a number of potential improvements to the report form to gain additional insights on failure conditions. For example, the future form will display different sets of questions to match operator role (Pilot in command, GCS operator, visual observer, etc.). Also, questions that were deemed too specific, such as the version of autopilot software and GCS software, will be removed.

\section{Conclusion}

The objective for the NASA-FAA UTM RTT C\&N subgroup is to explore operator solutions to ensure that UA are under operational control of the pilot and remain within a defined area. To support this exploration, off-nominal operational situation data, both digital and contextual, were collected in one of the UTM flight test events in 2017, the NC II, which involved the six FAA UAS test sites. The collected data were analyzed to inform future data collection improvements and to reinforce operational compliance. Whereas loss of navigation and loss of C2 link off-nominal situations were investigated with the collected digital data, significant filtering effort were necessary to identify correct set of data for the analysis. In addition, due to the low number of contextual off-nominal reports from the NC II, no significant findings trends were identified. For future testing, connecting findings from the digital data to contextual data to further increase insights into off-nominal operational situations has been suggested by safety experts. To realize this connection, improved mechanisms to gather, filter and validate digital data is being developed. Also, changes will be made to the future contextual data collection form to improve the amount and quality of the information. The next application of these improved evaluations of UA off-nominal conditions is planned for the next National Campaign in early 2018.

\section{Appendix A: FIMS-USS Architecture in the TCL2 UTM Research Platform}

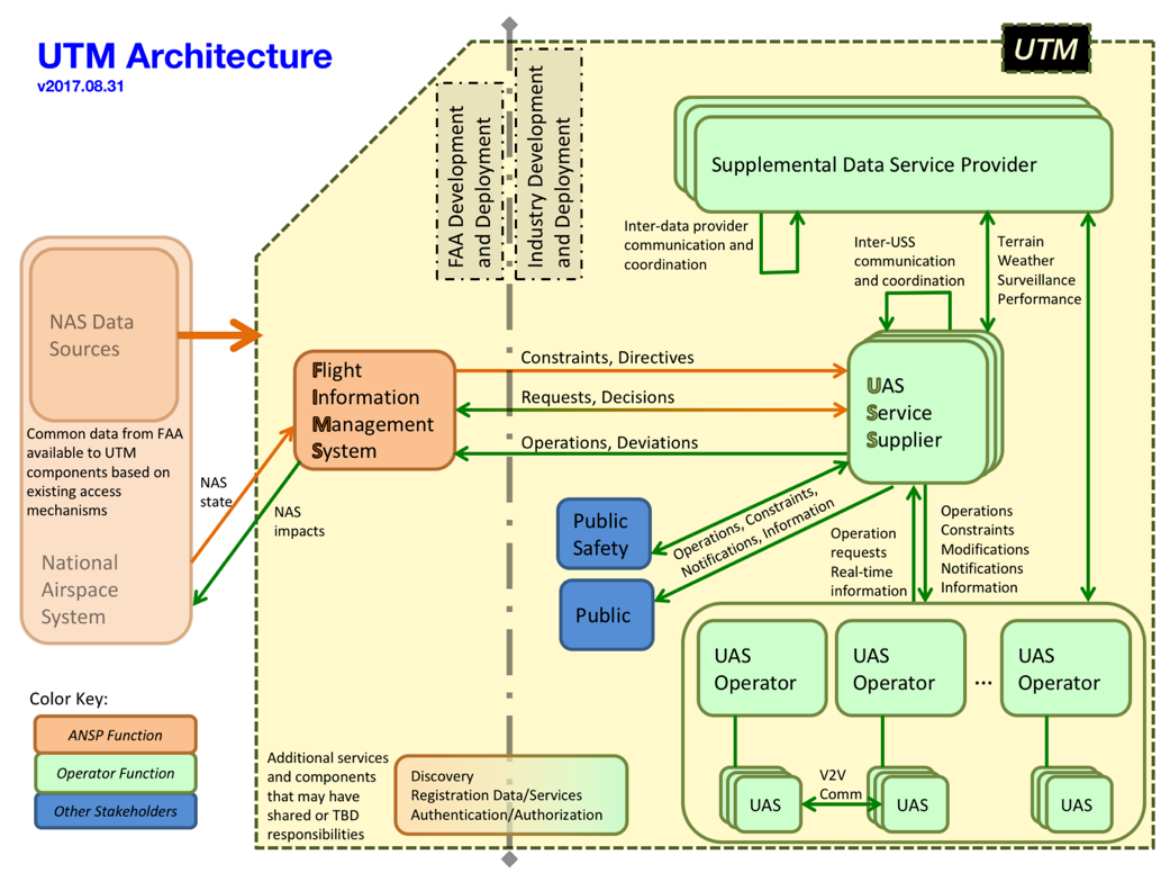




\section{Appendix B: The NC II DMP Elements}

Aircraft Flight Plan
\begin{tabular}{|l|l|l|}
\hline \multicolumn{1}{|c|}{ Variable Name } & \multicolumn{1}{c|}{ Type } & \multicolumn{1}{c|}{ Description } \\
\hline uvin & STRING & Version 4 UUID obtained from prototype NASA USS \\
\hline gufi & STRING & GUFI from the USS for this flight \\
\hline wpSequenceNum_nonDim & INTEGER & $\begin{array}{l}\text { Waypoint sequence number (i.e., sequence of waypoint for the aircraft to } \\
\text { fly to). Start with “0”. If no waypoints are available, use a single “-1". }\end{array}$ \\
\hline wpType_nonDim & INTEGER & Enter 1 for fly-over type, and 0 for fly-by type ${ }^{\dagger \dagger \dagger}$ \\
\hline wpLat_deg & FLOAT & Waypoint latitude (dec. degree), specify at least seven decimal degrees \\
\hline wpLon_deg & FLOAT & Waypoint longitude (dec. degree), specify at least seven decimal degrees \\
\hline wpAlt_ft & FLOAT & Waypoint altitude, WGS-84 (ft) \\
\hline wpTargetGroundSpeed_ftPerSec & FLOAT & Target ground speed at waypoint (ft/s) \\
\hline wpTargetAirSpeed_ftPerSec & FLOAT & Target airspeed at waypoint (ft/s) \\
\hline hoverTime_sec & FLOAT & $\begin{array}{l}\text { Time hovering at waypoint (if applicable) (sec). Include 3 decimal places } \\
\text { of precision. }\end{array}$ \\
\hline wpGpsWeek_wk & INTEGER & GPS Week arrival time at intended waypoint (if known) \\
\hline wpGpsSec_sec & FLOAT & $\begin{array}{l}\text { GPS SOW (seconds of week) arrival time at intended waypoint (if known). } \\
\text { Include 3 decimal places of precision. }\end{array}$ \\
\hline
\end{tabular}

\section{UAS Specifications}

\begin{tabular}{|c|c|c|}
\hline Variable Name & Type & Description \\
\hline uvin & STRING & Version 4 UUID obtained from prototype NASA USS \\
\hline aircraftReceiverSensitivity_dBm & FLOAT & Sensitivity of aircraft $\mathrm{C} 2$ receiver $(\mathrm{dBm})$ \\
\hline gcsReceiverSensitivity_dBm & FLOAT & Sensitivity of GCS receiver $(\mathrm{dBm})$ \\
\hline frequency_hz & FLOAT & The center frequency $(\mathrm{Hz})$ \\
\hline bandwidth $\mathrm{hz}$ & FLOAT & Bandwidth (Hz) \\
\hline protocol & STRING & e.g., MavLink, TCP/IP \\
\hline lateralNavPositionError95Pret_ft & FLOAT & $95 \%$ navigation system lateral error $(\mathrm{ft})$ \\
\hline verticalNavPositionError95Pret_ft & FLOAT & $95 \%$ navigation system vertical error $(\mathrm{ft})$ \\
\hline lateralNavVelocityError95Pret_ftPerSec & FLOAT & $95 \%$ navigation system lateral velocity error $(\mathrm{ft} / \mathrm{s})$ \\
\hline verticalNavVelocityError95Pret_ftPerSec & FLOAT & $95 \%$ navigation system vertical velocity error $(\mathrm{ft} / \mathrm{s})$ \\
\hline maxTakeoffWeightWithFuel_lb & FLOAT & $\begin{array}{l}\text { This is the empty weight of the vehicle plus energy source } \\
\text { (batteries or fuel) and the maximum payload the vehicle can carry } \\
\text { (lbs) }\end{array}$ \\
\hline numRotors_nonDim & INTEGER & $\begin{array}{l}\text { If vehicle is a multirotor or propeller driven aircraft, indicate the } \\
\text { number of rotors }\end{array}$ \\
\hline serviceCeiling_ft & INTEGER & Maximum altitude (MSL) that aircraft can nominally operate (ft) \\
\hline maxEndurance_sec & FLOAT & $\begin{array}{l}\text { This value corresponds to the endurance at maximum payload for } \\
\text { the given battery/power source(s) used by this vehicle (sec). } \\
\text { Include } 3 \text { decimal places of precision. }\end{array}$ \\
\hline maxRange_ft & FLOAT & $\begin{array}{l}\text { This value corresponds to the range at maximum payload for the } \\
\text { given battery/power source(s) used by this vehicle (ft) }\end{array}$ \\
\hline maxAirspeed ftPerSec & FLOAT & $(\mathrm{ft} / \mathrm{s})$ \\
\hline cruiseSpeed ftPerSec & FLOAT & $(\mathrm{ft} / \mathrm{s})$ \\
\hline maxRateOfAscent_ftPerSec & FLOAT & $(\mathrm{ft} / \mathrm{s})$ \\
\hline maxRateOfDescent_ftPerSec & FLOAT & $(\mathrm{ft} / \mathrm{s})$ \\
\hline minTurnRadius $\mathrm{ft}$ & FLOAT & $(\mathrm{ft})$ \\
\hline maxBankAngle_deg & FLOAT & (deg) \\
\hline maxTurnRate_degPerSec & FLOAT & $(\mathrm{deg} / \mathrm{s})$ \\
\hline maxPitchAngle_deg & FLOAT & $(\operatorname{deg})$ \\
\hline maxPitchRate_degPerSec & FLOAT & $(\mathrm{deg} / \mathrm{s})$ \\
\hline maxRollAngle_deg & FLOAT & (deg) \\
\hline maxRollRate degPerSec & FLOAT & $(\mathrm{deg} / \mathrm{s})$ \\
\hline maxYawAngle_deg & FLOAT & (deg) \\
\hline maxYawRate_degPerSec & FLOAT & $(\mathrm{deg} / \mathrm{s})$ \\
\hline
\end{tabular}

†† see http://tfmlearning.fly.faa.gov/Publications/atpubs/AIM/Chap1/aim0102.html for descriptions of fly-by and flyover waypoints. 


\begin{tabular}{|c|c|c|}
\hline payloadCapacity $\mathrm{lb}$ & FLOAT & (lb) \\
\hline maxGlideRatio nonDim & FLOAT & Glide ratio \\
\hline maxHeadWind_ftPerSec & FLOAT & $\begin{array}{l}\text { Headwind: applies to takeoff and landing only (i.e., not cruise } \\
\text { flight) }(\mathrm{ft} / \mathrm{s})\end{array}$ \\
\hline maxCrossWind_ftPerSec & FLOAT & $\begin{array}{l}\text { Crosswind: applies to takeoff and landing only (i.e., not cruise } \\
\text { flight) (ft/s) }\end{array}$ \\
\hline maxGust_ftPerSec & FLOAT & $(\mathrm{ft} / \mathrm{s})$ \\
\hline maxRpm_rotPerMin & FLOAT & For a single rotor (rpm) \\
\hline maxThrust lbf & FLOAT & Maximum thrust at cruise speed (lbf) \\
\hline maxAccel ftPerSec2 & FLOAT & Maximum Acceleration $\left(\mathrm{ft} / \mathrm{s}^{2}\right)$ \\
\hline posUpdateRate $\mathrm{hz}$ & FLOAT & Position update rate $(\mathrm{Hz})$ \\
\hline attUpdateRate hz & FLOAT & Vehicle orientation (attitude) update rate (Hz) \\
\hline timeDist2Command_sec & FLOAT & $\begin{array}{l}\text { Time from disturbance detection to command calculation } \\
\text { (human versus autopilot) }(\mathrm{sec}) \text {. Include } 3 \text { decimal places of } \\
\text { precision. }\end{array}$ \\
\hline timeCommand2Act_sec & FLOAT & $\begin{array}{l}\text { Time from command calculation to actuation (sec). Include } 3 \\
\text { decimal places of precision. }\end{array}$ \\
\hline jxx lbFt2 & FLOAT & Moment of inertia for $\mathrm{xx}\left(\mathrm{lb} \mathrm{ft}^{2}\right)$ \\
\hline jyy_lbFt2 & FLOAT & Moment of inertia for yy $\left(\mathrm{lb} \mathrm{ft}^{2}\right)$ \\
\hline jzz lbFt2 & FLOAT & Moment of inertia for $\mathrm{zz}\left(\mathrm{lb} \mathrm{ft}^{2}\right)$ \\
\hline jxy lbFt2 & FLOAT & Moment of inertia for xy $\left(\mathrm{lb} \mathrm{ft}^{2}\right)$ \\
\hline jxz lbFt2 & FLOAT & Moment of inertia for $\mathrm{xz}\left(\mathrm{lb} \mathrm{ft}^{2}\right)$ \\
\hline jyz lbFt2 & FLOAT & Moment of inertia for $\mathrm{yz}\left(\mathrm{lb} \mathrm{ft}^{2}\right)$ \\
\hline rotorInertia $\mathrm{lbFt} 2$ & FLOAT & For a single rotor $\left(\mathrm{lb} \mathrm{ft}^{2}\right)$ \\
\hline cl0_nonDim & FLOAT & $\begin{array}{l}\text { Lift coefficient for a fixed wing vehicle at steady-level flight trim } \\
\text { condition }\end{array}$ \\
\hline cd0_nonDim & FLOAT & $\begin{array}{l}\text { Drag coefficient for fixed wing or representative value for a multi } \\
\text { rotor at steady-level flight trim condition }\end{array}$ \\
\hline thrustCoefficient nonDim & FLOAT & Thrust coefficient for motor/rotor propelled vehicles \\
\hline kf_nonDim & FLOAT & $\begin{array}{l}\text { Force coefficient for multirotor motors. kf_nonDim*PWM }= \\
\text { Applied Force }\end{array}$ \\
\hline km_nonDim & FLOAT & $\begin{array}{l}\text { Force coefficients for multirotor motors. kf_nonDim*PWM = } \\
\text { Applied Force }\end{array}$ \\
\hline c2BerAircraft hz & FLOAT & Bit Error Rate at aircraft (bit errors per sec, in $\mathrm{Hz}$ ) \\
\hline c2BerGes_hz & FLOAT & Bit Error Rate at GCS (bit errors per sec, in Hz) \\
\hline
\end{tabular}

UAS State

Variable names for UAS state data (columns):

\begin{tabular}{|l|l|l|}
\hline Variable Name (columns) & Type & \multicolumn{1}{c|}{ Description } \\
\hline uvin & STRING & Version 4 UUID obtained from prototype NASA USS \\
\hline gufi & STRING & GUFI from the USS for this flight \\
\hline gpsWeek wk & FLOAT & GPS Week \\
\hline gpsSec_sec & FLOAT & GPS Time (SOW: seconds of week). Include 3 decimal places of precision. \\
\hline
\end{tabular}

Variable names for UAS state data (rows):
\begin{tabular}{|l|l|l|}
\hline \multicolumn{1}{|c|}{ Variable Name } & \multicolumn{1}{c|}{ Type } & \multicolumn{1}{c|}{ Description } \\
\hline vehiclePositionLat_deg & FLOAT & GPS Latitude (dec. deg) Report at least seven decimal degrees \\
\hline vehiclePositionLon_deg & FLOAT & $\begin{array}{l}\text { GPS Longitude (dec. deg) Report at least seven decimal } \\
\text { degrees }\end{array}$ \\
\hline vehiclePositionAlt_ft & FLOAT & Vehicle reported GPS altitude (ft) \\
\hline barometricAltitude_ft & FLOAT & Pressure based altitude (ft) \\
\hline barometricPressure_psi & FLOAT & Pressure sensor value used for barometric altitude (psi) \\
\hline altitudeUsedByAutopilot_ft & FLOAT & $\begin{array}{l}\text { Best estimate of altitude (filtered/processed) used by the } \\
\text { autopilot at any given time (ft) }\end{array}$ \\
\hline aboveTerrainAltitude_ft & FLOAT & $\begin{array}{l}\text { Best estimate of altitude above terrain (e.g. filtered/processed) } \\
(\mathrm{ft})\end{array}$ \\
\hline laserSensorAltitude_ft & FLOAT & Laser/LiDAR-sensor reported altitude above terrain (ft) \\
\hline opticalSensorAltitude_ft & FLOAT & $\begin{array}{l}\text { Optical/LED/infrared-sensor reported altitude above terrain } \\
(\mathrm{ft})\end{array}$ \\
\hline
\end{tabular}




\begin{tabular}{|c|c|c|}
\hline imageSensorAltitude_ft & FLOAT & $\begin{array}{l}\text { Camera/Image-processing-sensor reported altitude above } \\
\text { terrain (ft) }\end{array}$ \\
\hline radarSensorAltitude $\mathrm{ft}$ & FLOAT & Radar-sensor reported altitude above terrain (ft) \\
\hline acousticSensorAltitude $\mathrm{ft}$ & FLOAT & Sonar/ultrasonic-sensor reported altitude above terrain $(\mathrm{ft})$ \\
\hline indicatedAirspeed_ftPerSec & FLOAT & Indicated airspeed (ft/s) \\
\hline trueAirspeed ftPerSec & FLOAT & True airspeed $(\mathrm{ft} / \mathrm{s})$ \\
\hline groundSpeed ftPerSec & FLOAT & Ground Speed (ft/s) \\
\hline groundCourse deg & FLOAT & Ground Course (deg, True North) \\
\hline hdop_nonDim & FLOAT & HDOP: Horizontal dilution of precision of GPS constellation \\
\hline vdop_nonDim & FLOAT & VDOP: Vertical dilution of precision of GPS constellation \\
\hline numGpsSatellitesInView_nonDim & INTEGER & $\begin{array}{l}\text { Number of GPS satellites to which the aircraft has line-of-sight } \\
\text { to (whether acquired or not by the GPS receiver) }\end{array}$ \\
\hline numGpsSat nonDim & INTEGER & Number of GPS satellites tracked by GPS receiver \\
\hline roll deg & FLOAT & Roll (deg). Negative roll indicates left \\
\hline pitch_deg & FLOAT & Pitch (deg). Negative pitch indicates nose down \\
\hline yaw deg & FLOAT & Yaw (deg). Zero-degree yaw is North. \\
\hline velNorth_ftPerSec & FLOAT & Velocity-North (ft/s) \\
\hline velEast_ftPerSec & FLOAT & Velocity-East (ft/s) \\
\hline velDown_ftPerSec & FLOAT & Velocity-Down (ft/s) \\
\hline rollRate $\operatorname{degPerSec}$ & FLOAT & Roll Rate (deg/s) \\
\hline pitchRate_degPerSec & FLOAT & Pitch Rate (deg/s) \\
\hline yawRate degPerSec & FLOAT & Yaw Rate (deg/s) \\
\hline accBodyX ftPerSec2 & FLOAT & Acceleration-Body-x $\left(\mathrm{ft} / \mathrm{s}^{2}\right)$ \\
\hline accBodyY ftPerSec2 & FLOAT & Acceleration-Body-y $\left(\mathrm{ft} / \mathrm{s}^{2}\right)$ \\
\hline accBodyZ ftPerSec2 & FLOAT & Acceleration-Body- $\mathrm{z}\left(\mathrm{ft} / \mathrm{s}^{2}\right)$ \\
\hline motor1ControlThrottleCommand nonDim & FLOAT & \multirow{3}{*}{$\begin{array}{l}\text { Motor } 1-8 \text { control throttle command, between } 0 \% \text { and } 100 \% \text {. } \\
\text { If vehicle has a single engine, use motor } 1 \text { variable. }\end{array}$} \\
\hline motor2ControlThrottleCommand nonDim & FLOAT & \\
\hline motor3ControlThrottleCommand nonDim & FLOAT & \\
\hline motor4ControlThrottleCommand nonDim & FLOAT & \multirow{5}{*}{$\begin{array}{l}\text { For PWM, } 0 \%=\text { zero width duty cycle, } 100 \%=\text { full width duty } \\
\text { cycle, where } \\
\text { DutyCycle }=(\text { PulseWidth } / \text { Period }) \times 100 \% \text {. } \\
\text { For other motor control, use } 0 \%=\text { min.throttle setting, } \\
100 \%=\text { max.throttle setting }\end{array}$} \\
\hline motor5ControlThrottleCommand_nonDim & FLOAT & \\
\hline motor6ControlThrottleCommand nonDim & FLOAT & \\
\hline motor7ControlThrottleCommand nonDim & FLOAT & \\
\hline motor8ControlThrottleCommand_nonDim & FLOAT & \\
\hline aileronActuatorCommand_nonDim & FLOAT & $\begin{array}{l}-100 \% \text { to } 100 \% ; \text { where }-100 \%=\text { min. neg. deflection, } \\
100 \%=\text { max. positive deflection }\end{array}$ \\
\hline elevatorActuatorCommand_nonDim & FLOAT & $\begin{array}{l}-100 \% \text { to } 100 \% ; \text { where }-100 \%=\text { min. neg. deflection, } \\
100 \%=\text { max. positive deflection }\end{array}$ \\
\hline rudderActuatorCommand_nonDim & FLOAT & $\begin{array}{l}-100 \% \text { to } 100 \% ; \text { where }-100 \%=\text { min. neg. deflection, } \\
100 \%=\text { max. positive deflection }\end{array}$ \\
\hline flapActuatorCommand nonDim & FLOAT & 0 to $100 \% ;$ where $0 \%=$ fully retracted, $100 \%=$ fully extended \\
\hline landingGearActuatorCommand nondim & INTEGER & Either $0=$ Retracted or $1=$ Deployed \\
\hline batteryVoltage $\mathrm{v}$ & FLOAT & Vehicle Battery Voltage (V) \\
\hline batteryCurrent_a & FLOAT & Vehicle Battery Current (A) \\
\hline angleOfAttack deg & FLOAT & Angle of Attack (deg) \\
\hline sideSlip deg & FLOAT & Angle of Side Slip (deg) \\
\hline targetWaypointLat_deg & FLOAT & $\begin{array}{l}\text { Target waypoint latitude (dec. deg). Report at least seven } \\
\text { decimal degrees }\end{array}$ \\
\hline targetWaypointLon_deg & FLOAT & $\begin{array}{l}\text { Target waypoint longitude (dec. deg). Report at least seven } \\
\text { decimal degrees }\end{array}$ \\
\hline targetWaypointAlt $\mathrm{ft}$ & FLOAT & Target waypoint WGS-84 altitude (ft) \\
\hline aircraftControlMode_nonDim & INTEGER & $\begin{array}{l}\text { Aircraft control mode, specify an integer value for either } \\
\text { mode: } \text { Manual }=0 \text {, Automatic }=1 \text {, Mixed } \text { Mode }=2\end{array}$ \\
\hline targetGroundSpeed ftPerSec & FLOAT & Target vehicle ground speed $(\mathrm{ft} / \mathrm{s})$ \\
\hline targetAirSpeed ftPerSec & FLOAT & Target vehicle airspeed speed $(\mathrm{ft} / \mathrm{s})$ \\
\hline aircraftAirborneState_nonDim & INTEGER & $\begin{array}{l}\text { Real time report based on aircraft sensor. Specify an integer } \\
\text { value for current state: either Ground }=0 \text {, or Airborne }=1\end{array}$ \\
\hline
\end{tabular}




\begin{tabular}{|c|c|c|}
\hline minDistToDefinedAreaLateralBoundary_ft & FLOAT & $\begin{array}{l}\text { Orthogonal minimum lateral distance between UAS and the } \\
\text { boundary of the flight geography (defined area: allocated } \\
\text { airspace) (ft) }\end{array}$ \\
\hline minDistToDefinedAreaVerticalBoundary_ft & FLOAT & $\begin{array}{l}\text { Orthogonal minimum vertical distance between UA and the } \\
\text { boundary of the flight geography (defined area: allocated } \\
\text { airspace) (ft) }\end{array}$ \\
\hline c2RssiAircraft_dBm & FLOAT & C2 link RSSI measured in dBm at aircraft \\
\hline c2RssiGcs_dBm & FLOAT & C2 link RSSI measured in dBm at GCS \\
\hline c2NoiseAircraft_dBm & FLOAT & $\begin{array}{l}\text { Sum of Thermal noise power and RF interference power, } \\
\text { measured in } \mathrm{dBm} \text { at aircraft }\end{array}$ \\
\hline c2NoiseGcs_dBm & FLOAT & $\begin{array}{l}\text { Sum of Thermal noise power and RF interference power, } \\
\text { measured in } \mathrm{dBm} \text { at GCS }\end{array}$ \\
\hline c2PacketLossRateAircraftPrct_nonDim & FLOAT & Packet loss rate at aircraft ( 0 to $100 \%$ of packets lost) \\
\hline c2PacketLossRateGcsPret_nonDim & FLOAT & Packet loss rate at GCS ( 0 to $100 \%$ of packets lost) \\
\hline lateralNavPositionError_ft & FLOAT & Current navigation system lateral error (ft) \\
\hline verticalNavPositionError_ft & FLOAT & Current navigation system vertical error (ft) \\
\hline lateralNavVelocityError_ftPerSec & FLOAT & Current navigation system lateral velocity error $(\mathrm{ft} / \mathrm{s})$ \\
\hline verticalNavVelocityError_ftPerSec & FLOAT & Current navigation system vertical velocity error $(\mathrm{ft} / \mathrm{s})$ \\
\hline
\end{tabular}

\section{Auxiliary UAS Operation}

Variable names for auxiliary UAS operation data (columns):

\begin{tabular}{|l|l|l|}
\hline Variable Name (columns) & \multicolumn{1}{|c|}{ Type } & \multicolumn{1}{c|}{ Description } \\
\hline uvin & STRING & Version 4 UUID obtained from prototype NASA USS \\
\hline gufi & STRING & GUFI from the USS for this flight \\
\hline
\end{tabular}

Variable names for auxiliary UAS operation data (rows):

\begin{tabular}{|l|l|l|}
\hline \multicolumn{1}{|c|}{ Variable Name (rows) } & \multicolumn{1}{c|}{ Type } & \multicolumn{1}{c|}{ Description } \\
\hline typeOfOperation & STRING & Use either "Live", Simulated" or "Test" \\
\hline flightTestCardName & STRING & $\begin{array}{l}\text { Name of the flight test card. 280 characters maximum, between quote marks " ", } \\
\text { all characters in between are valid except for quote marks as they signal the } \\
\text { beginning and end of the string. }\end{array}$ \\
\hline takeOffWeight_lb & FLOAT & Vehicle weight at takeoff (lb) \\
\hline takeOffTimeGpsWeek_wk & INTEGER & $\begin{array}{l}\text { GPS time (week) of takeoff, defined as the moment the vehicle leaves the } \\
\text { ground/launching device }\end{array}$ \\
\hline takeOffTimeGpsSec_sec & FLOAT & $\begin{array}{l}\text { GPS time (SOW: seconds of week) of takeoff, defined as the moment the vehicle } \\
\text { leaves the ground/launching device. Include 3 decimal places of precision. }\end{array}$ \\
\hline takeoffPosLat_deg & FLOAT & Takeoff position latitude (dec. degree). Report at least seven decimal degrees \\
\hline takeoffPosLon_deg & FLOAT & Takeoff position longitude (dec. degree). Report at least seven decimal degrees \\
\hline takeoffPosAlt_ft & FLOAT & Takeoff position WGS-84 altitude (ft) \\
\hline landingPosLat_deg & FLOAT & $\begin{array}{l}\text { Landing/recovery position latitude (dec. degree). Report at least seven decimal } \\
\text { degrees }\end{array}$ \\
\hline landingPosLon_deg & FLOAT & $\begin{array}{l}\text { Landing/recovery position longitude (dec. degree). Report at least seven decimal } \\
\text { degrees }\end{array}$ \\
\hline landingPosAlt_ft & FLOAT & Landing/recovery position WGS-84 altitude (ft) \\
\hline gcsPosLat_deg & FLOAT & $\begin{array}{l}\text { Ground Control System (GCS) position latitude (dec. degree). Report at least } \\
\text { seven decimal degrees }\end{array}$ \\
\hline gcsPosLon_deg & FLOAT & GCS position longitude (dec. degree). Report at least seven decimal degrees \\
\hline gcsPosAlt_ft & FLOAT & GCS position WGS-84 altitude (ft) \\
\hline
\end{tabular}

\section{Appendix C: Questions in the NC II Online Off-nominal Report Form}

\section{If you were the Pilot In Command (PIC), were you the...}

O RC Pilot

O GCS Operator

2. If you were NOT the PIC, what was your role?

O Payload System Operator

O Range Safety Officer (RSO)

O Visual Observer 
O Mission Manager

Principal Investigator (PI)

$\bigcirc$ Test Site Coordinator

O Other:

3. What was the Test Site Location

University of Alaska - Alaska

O NIAS - Nevada

Griffiss - New York

O NPUASTS - North Dakota

Lone Star UAS Center - Texas

O Mid Atlantic Partnership - Virginia

4. What was GCS Launch Location/Identification (as used in the Flight Plan); e.g. GCS-2, etc.

5. What were the environmental conditions at the location and time of the event?

Wind

Wind Direction:

Wind Speed: (degrees magnetic)

Location of weather measurement: (in knots)

6. At what time were these measurements taken (local time)?

7. What was the density altitude (calculated in feet MSL) at the location and time of the event?

Density altitude is the indicated altitude adjusted for the barometric setting (e.g., 29.92"), and outside air temperature (e.g., 30 degrees C.) For example, an aircraft at 6,000 feet MSL, with an altimeter setting of 29.89 inches of mercury, at an outside air temperature of $30^{\circ}$ Centigrade $\left(86^{\circ}\right.$ Fahrenheit) is operating at a density altitude of 8,020 feet.

8. What are the Aircraft \& Associated Control Systems?

FAA Vehicle Registration (e.g., N65SR, or FA number, etc.)

Vehicle Make \& Model

Autopilot Make \& Model

Autopilot Software Version

Ground Control Station Software Program

Ground Control Station Software Version

Flight hours on platform

Time since last maintenance

9. What Communications Frequencies were in use for GCS, RC Controller, and Payload?

10. Are you aware of any frequency interference due to communication radio or cellphone use in proximity to the GCS at the time of the event? Explain if any.

11. Was the flight within Visual Line Of Sight (VLOS), or Beyond Visual Line Of Sight (BVLOS)?

12. What was the Date and Time at the time of Launch? (MM/DD/YYYY - hh:mm local)

13. What was the distance of the vehicle from the launch site of the time of the event? (distance in feet)

14. Which of the following occurred?

$\square$ Loss/Degradation of vehicle to GCS communication

Loss/Degradation of GCS to vehicle communication

GPS Satellite or other navigation system signal loss/degradation

Other navigation system failure

Lateral Deviation from flight geography

Vertical Deviation from flight geography

15. What Navigation Geofence Limitation was Triggered or Breached?

Lateral Geofence

Vertical Geofence (climb or descent above/below flight geography)

No Geofence Enabled

None

16. What Navigation Geofence SYSTEM was Triggered or Breached?

$\square$ Vehicle Geofence

GCS Geofence

Not Applicable

17. What Loss of Aircraft Control Occurred

Power (battery) failure

Motor failure

Propeller Failure

Flight Control System failure 
Structural failure

Other (please specify)

18. What Action was Taken and what was the Result?

Regained Communication

Regained Navigation Capability

Regained Aircraft Control

Flight Returned to Planned Track or Heading

$\square$ Return to flight geography (authorized airspace)

$\square$ Return To Base (Return To Launch Site)

Land In Place

Loiter

Terminate

Vehicle lost

Vehicle impacted terrain

None

Other (please specify)

\section{Section 8 - Reporter Narrative}

Keeping in mind the topics shown below, discuss those that you feel are relevant and anything else you think is important. Include what you believe really caused the problem, and what can be done to prevent a recurrence, or correct the situation.

\section{CHAIN OF EVENTS}

How the problem arose $\bullet$ How it was discovered $\bullet$ Contributing factors $\bullet$ Corrective actions

HUMAN PERFORMANCE CONSIDERATIONS

Perceptions, judgments, decisions • Actions or inactions • Factors affecting the quality of human performance

\section{Acknowledgement}

F. A. Authors thank the UTM project systems engineering team, software team and AOL persons for their support in ingesting and validating the NC II digital data for analysis.

\section{References}

[1] FAA Aerospace Forecast Fiscal Years 2017-2037, Publication, FAA, Washington, D.C., 2016.

[2] Small Unmanned Aircraft Rule, The Federal Aviation Regulation (FAR) Part 10, FAA, Washington, D.C., 2016.

[3] Request a Part 107 Waiver or Operation in Controlled Airspace [online], URL: https://www.faa.gov/uas/request waiver/ [accessed 24 October 2017].

[4] Kopardekar, P., Rios, J., Prevot, T., Johnson, M., Jung, J., and Robinson, J. E., "Unmanned Aircraft System Traffic Management (UTM) Concept of Operations," 16th AIAA Aviation Technology, Integration, and Operations Conference, Washington, D.C., 13-17 June 2016.

[5] Kopardekar, P., "Unmanned Aerial System Traffic Management (UTM): Enabling Low-altitude Airspace and UAS Operations,” Tech. Rep. NASA TM-2014-21829, 2014.

[6] UAS Traffic Management (UTM) Research Transition Team (RTT) Plan, Publication, FAA, Washington, D.C., 2017.

[7] The FAA UAS Test Sites [online], URL: https://www.faa.gov/uas/research/test sites/ [accessed 13 April 2018 ].

[8] Aweiss, A., Owens, D., Rios, J., Homola, J, and Mohlenbrink, C., "Unmanned Aircraft Systems (UAS) Traffic Management (UTM) National Campaign II,” AIAA SciTech Forum, Kissimmee, FL, 8-12 January 2018.

[9] Aviation Safety Information Analysis and Sharing [online], URL: https://portal.asias.aero/web/guest [accessed 24 October 2017].

[10] Halford, C. and Harper, M., “ASIAS: Aviation Safety Information Analysis and Sharing”, 27th IEEE/AIAA Digital Avionics systems Conference, St. Paul, Minnesota, 26-30 October 2008.

[11] NASA Aviation Safety Reporting System [online], URL: https://asrs.arc.nasa.gov/ [accessed 24 October 2017].

[12] Withrow, C. A. and Reveley, M. S., "Analysis of Aviation Safety Reporting System Incident Data Associated with the Technical Challenges of the System-Wide Safety and Assurance Technologies Project”, Publication, NASA/TM-2015-217899, NASA, Washington, D.C., 2015.

[13] ASRS: The Case for Confidential Incident Reporting Systems, Publication, NASA, Washington, D.C., 2001. 